\title{
Chinese ASCVD risk equations rather than pooled cohort equations are better to identify macro- and microcirculation abnormalities
}

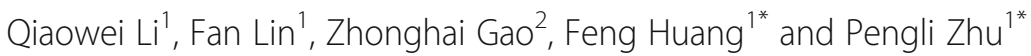

\begin{abstract}
Background: We hypothesized that discriminating the early subclinical organ damage would serve as a great opportunity for prevention against atherosclerotic cardiovascular disease (ASCVD). Brachial-ankle pulse wave velocity (baPWV), low retinal vascular fractal dimension, and albuminuria are surrogates of subclinical vascular changes.

Methods: The aim of this study was to use Pooled Cohort Equations (PCE) and ASCVD risk equations derived from "Prediction for ASCVD Risk in China project (CHINA-PAR)" to observe the prevalence of macro- and microcirculation abnormalities. A total of 2166 subjects were involved. Characteristics were investigated using questionnaire and physical examinations. We calculated the urine albumin to creatinine ratio (UACR). The baPW was measured using a fully automatic arteriosclerosis detector. The retinal vascular fractal dimension was measured by a semiautomated computer-based program. The 10-year ASCVD risk was estimated using the PCE and CHINA-PAR model.

Results: The cut-off values for the elevated baPWV were 2.82 and $2.92 \%$ in the PCE model and CHINA-PAR model, respectively, with nearly $85 \%$ sensitivity and an average specificity of $74 \%$. For low retinal fractal dimension, at the cut-off point of $3.8 \%$, we acquired an acceptable sensitivity of $66.27-68.24 \%$ and specificity of $62.57-67.45 \%$. All the $C$-statistics presented a significant improvement from the PCE model to the CHINA-PAR model $(P<0.05)$. For all categories-net reclassification improvement (NRI) values were significant and clearly varied $(0.329,0.183$, and 0.104, respectively) depending on the cut-off set at 3\%.

Conclusion: Our study demonstrated that the CHINA-PAR equations rather than PCE could provide better identification of macro- and microcirculation abnormalities. A lower cut-off point for the subclinical vascular changes may be selected in a population from southeast China.
\end{abstract}

Keywords: ASCVD, Macrocirculation, Microcirculation

* Correspondence: wmhf0327@126.com; zpl7755@hotmail.com

${ }^{1}$ Department of Geriatric Medicine, Fujian Provincial Hospital, Fujian

Provincial Center for Geriatrics, Fujian Provincial Key Laboratory of Geriatric

Disease, Shengli Clinical Medical College of Fujian Medical University, 134

East Street, Fuzhou 350001, Fujian, China

Full list of author information is available at the end of the article

C C The Author(s). 2020 Open Access This article is licensed under a Creative Commons Attribution 4.0 International License, which permits use, sharing, adaptation, distribution and reproduction in any medium or format, as long as you give appropriate credit to the original author(s) and the source, provide a link to the Creative Commons licence, and indicate if changes were made. The images or other third party material in this article are included in the article's Creative Commons licence, unless indicated otherwise in a credit line to the material. If material is not included in the article's Creative Commons licence and your intended use is not permitted by statutory regulation or exceeds the permitted use, you will need to obtain permission directly from the copyright holder. To view a copy of this licence, visit http://creativecommons.org/licenses/by/4.0/. The Creative Commons Public Domain Dedication waiver (http://creativecommons.org/publicdomain/zero/1.0/) applies to the data made available in this article, unless otherwise stated in a credit line to the data. 


\section{Background}

Cardiovascular disease (CVD) produces severe health and economic burden in China and globally $[1,2]$. There were an estimated 93.8 million cases of CVD overall during 2016 in China, which is more than twice the cases that were reported in 1990 [1]. For better prevention of CVD and individualized therapy, several risk factors have been established. In 2013, the American College of Cardiology and the American Heart Association developed the Pooled Cohort Equations (PCE) to estimate the 10-year risk of developing a first hard atherosclerotic CVD (ASCVD) event (defined as the first occurrence of nonfatal myocardial infarction, coronary heart disease, death, or stroke) [3]. Due to ethnic differences, estimated risks may be over-estimated in Asian-American populations in the PCE model. A new tool to conduct ASCVD risk prediction called the Prediction for ASCVD Risk in China project (CHINA-PAR) was subsequently developed in 2016 [4]. The CHINA-PAR equations are based on traditional major risk factors with several new variables, validated by 4 Chinese cohorts, and aimed to serve as valuable predictor of ASCVD risk in the general Chinese population. However, studies have shown controversial results with the CHINA-PAR model. It outperformed PCE in ASCVD risk prediction in a rural northern Chinese population [5] but underestimated the risk in Mongolians [6]. Thus, further validation of ASCVD risk predictions in other Chinese populations needs to be performed.

ASCVD has a latency of many years and produces multiple subclinical manifestations during its progression. Therefore, we hypothesized that discriminating the early macro- and microvascular subclinical organ damage would provide a great opportunity to develop preventative interventions. In the macrocirculation, the measurement of arterial stiffness parameters was performed by brachial-ankle pulse wave velocity (baPWV) to predict the risk of future CV events and total mortality, which is recommended by the guidelines for the management of hypertension in Japan [7]. In the microcirculation, low retinal vascular fractal dimension indicates reduced complexity in the branching pattern of the microvasculature, independent of stroke risk factors [8], and albuminuria is associated with retinopathy, stroke, heart failure, and atherosclerosis [9-12].

Although these methods for early organ damage above are noninvasive, the technical complexity and time consumption may reduce the detection rate. Therefore, we aimed to use the ASCVD risk scores to observe the prevalence of macro- and microcirculation abnormalities in a Chinese population, achieving a more valuable equation by comparing the PCE and CHINA-PAR models.

\section{Methods}

\section{Subjects}

Our study was based on a cross-sectional study of hypertension conducted from July 2011 to November 2011. A total of 7 coastal villages in Fujian Province were randomly selected in cluster sampling. Voluntary participants aged 30 years or more living in the above selected areas for more than 5 years were enrolled. Subjects without complete data or qualified fundus photographs were excluded. We also excluded subjects previously diagnosed with ASCVD (including myocardial infarction, coronary heart disease and stroke) or with a suspicion of active inflammatory disease. Two thousand one hundred sixty-six subjects were involved finally (Fig. 1). This study was performed according to the World Medical Association Declaration of Helsinki and approved by the Ethics Committee of the Fujian Provincial Hospital. A written informed consent was obtained from all individuals.

\section{Data collection}

Questionnaires were administered to collect following variables: age, smoking habits, alcohol consumption, medical history (including hypertension, diabetes, myocardial infarction, cardiomyopathy, congenital heart disease, peripheral vascular disease, congestive heart failure, stroke, acute and chronic inflammatory diseases, malignancy, and hematological disorders), and medications. Participants underwent physical examinations to measure height, weight, waist circumference, hip circumference, and blood pressure. Body mass index (BMI) was calculated as the weight $(\mathrm{kg}) /$ height $^{2}\left(\mathrm{~m}^{2}\right)$. Fasting blood samples were collected for laboratory testing including total cholesterol (TC), high-density lipoprotein cholesterol (HDL-C), low-density lipoprotein cholesterol (LDL-C), glycosylated hemoglobin (HbA1c), and serum creatinine. Urinary creatinine and albumin were measured from the morning urine sample. We calculated estimated glomerular filtration rate (eGFR) with the Chronic Kidney Disease Epidemiology Collaboration equation [13]. The urine albumin to creatinine ratio (UACR) was defined as the ratio of urine albumin to urine creatinine [14]. The subjects who had been diagnosed or already took related medication were defined as hypertensive [15]. Participants with a clear history, or were currently taking anti-diabetic medications, or with an $\mathrm{HbA} 1 \mathrm{c} \geq 6.5 \%$ were defined as diabetic [16].

\section{Measurement of baPWV}

After resting for more than $10 \mathrm{~min}$, the baPWV was measured using a fully automatic arteriosclerosis detector (Colin VP-1000 device; Colin Medical Technology Company, Komaki, Japan) by two professionals. We selected the right baPWV for analysis. The inter- and 


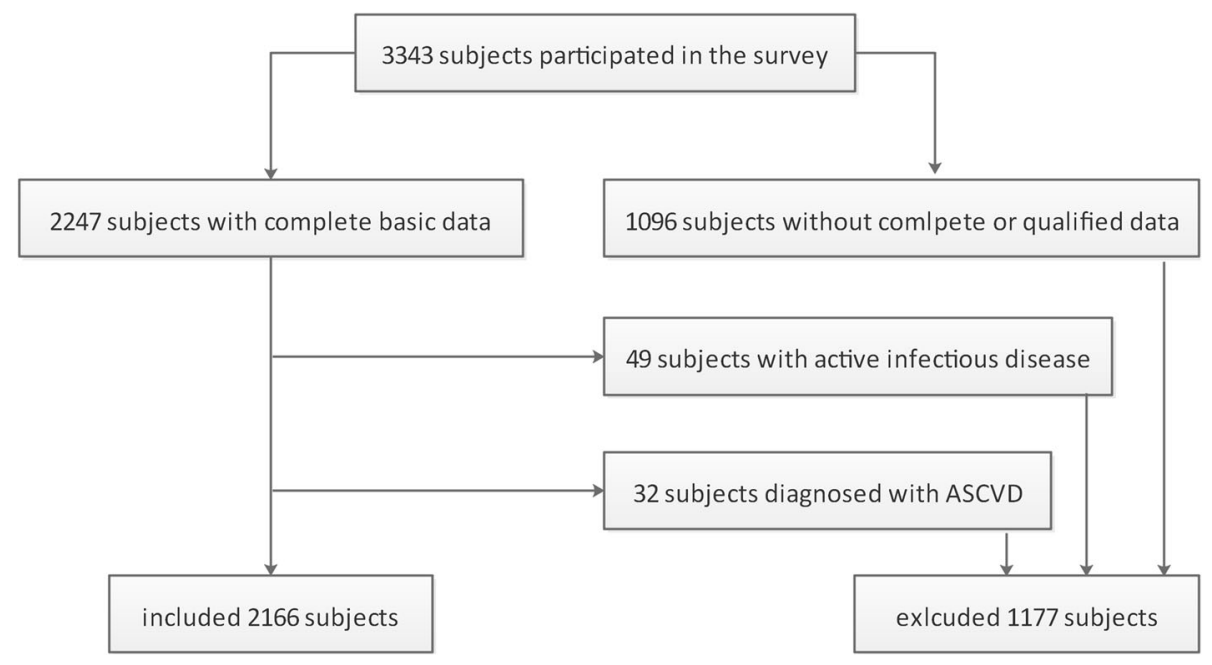

Fig. 1 Flowchart of the study population. ASCVD, atherosclerotic cardiovascular disease

intra-observer variability coefficients were 0.93 (95\% CI: $0.92-0.93$ ) and 0.99 (95\% CI: 0.98-0.99), respectively.

\section{Retinal fractal dimension}

For documentation of non-mydriatic fundus photographs, two ophthalmologists used digital cameras (Topcon NW8, TOPCON CORPORATION, Tokyo, Japan, and Nikon D90, Tokyo, Japan) centering on the optic disk with a capturing range of $45^{\circ}$. A semiautomated computer-based program (Singapore I Vessel Assessment version 3.0 software, jointly developed by Singapore National University and Singapore Eye Research Institute, Singapore) was used to measure retinal vascular fractal dimension in the range of 0.5-2 DD from the disc margin. In a random sample of 100 fundus photographs, we found no differences between intra-grader measurements. The intergrader correlation coefficient for Df was 0.90 (95\% CI: 0.89-0.90).

\section{Assessment of ASCVD risk}

The 10-year ASCVD risk was estimated using the PCE model recommended by the 2013 American College of Cardiology/American Heart Association (ACC/AHA) [3] and the CHINA-PAR model produced based on a Chinese

population [4], respectively. The basic equation is 1-

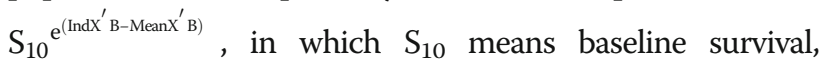
lndX'B is calculated by sum of "Coeffcient $\times$ Value", and MeanX'B stands for mean of "Coeffcient $\times$ Value" (details showed in Supplemental Table 1). The PCE included gender, age, race, diagnosis of diabetes, smoking, treated hypertension, systolic blood pressure, and lipid profile values (TC and HDL-C) as variables. The CHINA-PAR equations contained the major risk factors adopted in the PCE and additional variables including waist circumference, geographic region (northern vs. southern), urbanization (urban vs. rural), and family history of ASCVD.

\section{Definition of macro- and microcirculation abnormalities}

The cut-off value for the normal and increased baPWV was $1400 \mathrm{~cm} / \mathrm{s}$ [17]. The lowest quartile of the retinal vascular fractal dimension was defined as a low fractal dimension. Albuminuria was defined as UACR $\geq 30 \mathrm{mg} / \mathrm{g}$ [14].

\section{Statistical analysis}

All statistical analyses were conducted using $\mathrm{R}$ version 3.5.3 and $\mathrm{MedCalc}^{\circ}$ statistical software, and a $P$-value < 0.05 was considered statistically significant. Normally distributed continuous data are given as means \pm SDs, skewed distributed continuous data are reported as median (25th, 75th percentiles), categorical variables are presented as the percentage of patients. Receiver operating characteristic (ROC) curves were applied to evaluate macro- and microcirculation abnormalities. Cut-off points were determined according to the Youden index with the best-combined sensitivity and specificity. Prognostic comparisons between models were done using Harrell's concordance C-statistic. We explored categorical net reclassification improvement (NRI) to evaluate the stratification of effects from the PCE model to the CHINA-PAR model at the cut-off point derived from the ROC.

\section{Results}

Table 1 shows characteristics of the study population. A total of 2166 individuals were enrolled in this study, with an average age of $51.91 \pm 11.91$ years and $37.4 \%$ men. The median UACR was $10.17 \mathrm{mg} / \mathrm{g}$ (interquartile range $4.13-22.98 \mathrm{mg} / \mathrm{g}$ ). The mean retinal fractal dimension was $1.37 \pm 0.05$. The median baPWV for all subjects was 
Table 1 Characteristics of study population

\begin{tabular}{|c|c|}
\hline subjects, n & 2166 \\
\hline age, years & $51.91 \pm 11.91$ \\
\hline systolic BP, mmHg & $126.89 \pm 21.90$ \\
\hline diastolic BP, mmHg & $78.53 \pm 11.84$ \\
\hline WHR & $0.85 \pm 0.07$ \\
\hline $\mathrm{BMI}, \mathrm{kg} / \mathrm{m}^{2}$ & $23.83 \pm 3.42$ \\
\hline total cholesterol, mmol/L & $5.03 \pm 1.05$ \\
\hline $\mathrm{HDL}-\mathrm{C}, \mathrm{mmol} / \mathrm{L}$ & $1.23 \pm 0.33$ \\
\hline LDL-C, mmol/L & $2.78 \pm 0.88$ \\
\hline $\mathrm{eGFR}, \mathrm{ml} / \mathrm{min} / 1.73 \mathrm{~m}^{2}$ & $103.25 \pm 33.62$ \\
\hline $\mathrm{HbA1c}, \%$ & $5.72 \pm 0.68$ \\
\hline UACR, mg/g & $10.17(4.13,22.98)$ \\
\hline fractal dimension & $1.37 \pm 0.05$ \\
\hline $\mathrm{baPW}, \mathrm{cm} / \mathrm{s}$ & $1346(1170,1600)$ \\
\hline male, n (\%) & $810(37.4)$ \\
\hline hypertension history, n (\%) & $512(23.6)$ \\
\hline diabetes history, n (\%) & 185(8.5) \\
\hline smoking, n (\%) & $414(19.1)$ \\
\hline drinking, n (\%) & $339(15.7)$ \\
\hline \multicolumn{2}{|c|}{$\begin{array}{l}\text { Normally distributed continuous data are given as mean } \pm \text { SD, skewed } \\
\text { distributed continuous data are reported as median ( } 25 \text { th, } 75 \text { th percentiles), } \\
\text { categorical variables are presented as a percentage of patients. BP Blood } \\
\text { pressure, WHR Waist-hip rate, BMI Body mass index, HDL-C High density } \\
\text { lipoprotein-cholesterol, LDL-C Low density lipoprotein-cholesterol, eGFR } \\
\text { Estimated glomerular filtration rate, HbA1c Glycated haemoglobin A1c, UACR } \\
\text { Urine albumin-to-creatinine ratio, baPWV Brachial-ankle pulse wave velocity }\end{array}$} \\
\hline
\end{tabular}

$1346 \mathrm{~cm} / \mathrm{s}$, with an interquartile range of $1170-1600 \mathrm{~cm} / \mathrm{s}$. The prevalence of hypertension, diabetes mellitus, smoking, and drinking were 23.6, 8.5, 19.1, and 15.7\%, respectively.

Cut-off values, sensitivity, and specificity with the highest Youden index are shown in Table 2. The cut-off values for the elevated baPWV were 2.82 and $2.92 \%$ in

Table 2 Highest Youden index of macro- and microcirculation abnormalities and corresponding cut-off, sensitivity, and specificity

\begin{tabular}{|c|c|c|c|c|}
\hline risk scores & Youden index & cut-off & sensitivity & specificity \\
\hline \multicolumn{5}{|c|}{ elevated baPWV } \\
\hline PCE & 55.97 & 2.82 & 84.14 & 71.84 \\
\hline CHINA-PAR & 62.18 & 2.92 & 84.25 & 77.93 \\
\hline \multicolumn{5}{|c|}{ low fractal dimension } \\
\hline PCE & 30.81 & 3.79 & 68.24 & 62.57 \\
\hline CHINA-PAR & 33.72 & 3.77 & 66.27 & 67.45 \\
\hline \multicolumn{5}{|l|}{ albuminuria } \\
\hline PCE & 15.00 & 11.09 & 35.15 & 79.85 \\
\hline CHINA-PAR & 18.52 & 3.06 & 61.14 & 57.38 \\
\hline
\end{tabular}

Values are presented as \%. baPWV Brachial-ankle pulse wave velocity, PCE Pooled cohort equation recommended by the 2013 American College of Cardiology and American Heart Association guidelines; CHINA-PAR, equations for 10-year ASCVD risk prediction in Chinese populations the PCE model and the CHINA-PAR model, respectively, with nearly $85 \%$ sensitivity and an average specificity of $74 \%$. For the low retinal fractal dimension, at the cut-off point of $3.8 \%$, we achieved an acceptable sensitivity of $66.27-68.24 \%$ and specificity of $62.57-67.45 \%$. However, for albuminuria, neither the PCE model nor the CHINA-PAR model would achieve sensitivity higher than $61.14 \%$. In this case, we set a new cut-off point at $3 \%$ for the high-risk category (PCE and CHINA-PAR models) for reclassification.

The area under the ROC curve calculated for discriminating elevated baPWV was 0.86 for PCE (95\% CI: 0.84-0.87) and 0.88 for CHINA-PAR (95\% CI: 0.870.90). To distinguish the low fractal dimension, the Cstatistics were 0.71 (95\% CI: 0.69-0.73) for PCE and 0.72 (95\% CI: 0.70-0.74) for CHINA-PAR. The C-statistics to detect albuminuria were 0.59 (95\% CI: 0.57-0.61) for PCE and 0.61 (95\% CI: 0.59-0.63) for CHINA-PAR. All the $\mathrm{C}$-statistics presented a significant improvement from the PCE model to the CHINA-PAR model $(P<$ $0.05)$. All categorical-NRI values were significant and varied across groups $(0.329,0.183$, and 0.104 , respectively) depending on the cut-off set at $3 \%$. (Table 3 and Fig. 2).

\section{Discussion}

To our knowledge, our study is the first to validate the incremental value of CHINA-PAR equations against PCE for discriminating macro- and microcirculation abnormalities.

In this cross-sectional study, we assessed the performance of the PCE and CHINA-PAR models for macro- and microcirculation abnormalities in a southern Chinese rural population.

Our results showed that both models showed acceptable discrimination of elevated baPWV and low retinal vascular fractal dimension. Compared to PCE, CHINAPAR equations showed a significant improvement in the prevalence stratification of macro- and microcirculation abnormalities represented by elevated baPWV, low retinal vascular fractal dimension, and UACR. After resetting the cut-off for the high-risk group at $3 \%$, the prevalence of the reclassification was significantly better in the CHINA-PAR equations than in the PCE.

Subclinical vascular changes both in macro- and microcirculation would progress in ASCVD.

As a representative marker of macro-circulation, arterial stiffness measured with the baPWV has the ability to predict future $\mathrm{CV}$ events and total mortality [7]. Although ageing exerts a strong influence on the baPWV [18], the reference values for the baPWV and Asian evidence showed $1400 \mathrm{~cm} / \mathrm{s}$ is an appropriate cutoff $[17,19]$. From the microcirculation perspective, retinal microvasculopathy may reflect small vessel disease. 
Table 3 Comparisons of PCE and CHINA-PAR with a difference in AUC and NRI for prediction of macro- and microcirculation abnormalities

\begin{tabular}{lllllll}
\hline & C-statistics of PCE model $(95 \% \mathrm{Cl})$ & $\begin{array}{l}\text { C-statistics of CHINA-PAR } \\
\text { model }(95 \% \mathrm{Cl})\end{array}$ & $\Delta \mathrm{AUC}(95 \% \mathrm{Cl})$ & $P$ & categorical NRI $(95 \% \mathrm{Cl})$ & $P$ \\
\hline elevated baPWV & $0.86(0.84-0.87)$ & $0.88(0.87-0.90)$ & $0.028(0.020-0.036)$ & $<0.001$ & $0.329(0.290,0.368)$ & $<0.001$ \\
low fractal dimension & $0.71(0.69-0.73)$ & $0.72(0.70-0.74)$ & $0.012(0.002-0.022)$ & 0.019 & $0.183(0.137,0.228)$ & $<0.001$ \\
albuminuria & $0.59(0.57-0.61)$ & $0.61(0.59-0.63)$ & $0.016(0.005-0.027)$ & 0.032 & $0.104(0.053,0.156)$ & $<0.001$ \\
\hline
\end{tabular}

Cut-off for categorical NRI is 3.00\%. PCE, pooled cohort equation recommended by the 2013 American College of Cardiology and American Heart Association guidelines; CHINA-PAR-equations for 10-year ASCVD risk prediction in Chinese populations; AUC Area under the receiver operative curve (C-statistics); CI Confidence interval, NRI Net reclassification improvement; baPWV brachial-ankle pulse wave velocity

The lower fractal dimension which reflects a sparser retinal microvascular network is associated with stroke, Alzheimer's disease, and more diffuse and severe coronary artery disease [20-23]. As demonstrated, albuminuria is significantly and independently associated with the presence and severity of atherosclerosis [12]. Therefore, we considered elevated baPWV, lower fractal dimension, and albuminuria proxies of macro- and microcirculation abnormalities.

Matthew et al. indicated that the PCE risk score provided a better estimate of racial differences in vascular function and structure than the Framingham risk score did [24]. Few former studies have assessed the association of ASCVD risk by CHINA-PAR equations with subclinical vascular changes. It may help to identify Chinese individuals at earlier time points who could benefit from early commencement or strengthening of medical treatment. The finding in the current study supports that the CHINA-PAR equations provides utility in identifying patients with elevated baPWV and low fractal dimension, in addition to a significantly improved risk assessment of all the macro- and microcirculation abnormalities as compared to PCE.

Since the CHINA-PAR equations were developed in 2016 , the validations in real practice mostly focused on the northern population $[5,6]$. To date, an approximately 6-fold difference in the total burden of CVD persists among provinces [1]; therefore, geographical strategies are needed to identify ASCVD throughout China in specific provinces. Our study proposed a lower cut-off point at $3 \%$ to classify individuals at high-risk for macro- and microcirculation abnormalities. The discrepancy may be attributed to the lowest rates for ASCVD in China being in the Fujian province and their dietary difference with a higher intake of proteins, unsaturated fatty acids, magnesium, zinc, and calcium. Our study also underscored that it is crucial to pay attention to those who belong to the low-risk group but as a result of the reclassification by the adjusted cut-off point, were then reclassified as high-risk, and therefore require more intense therapy.

Strengths of our study include three parameters to indicate subclinical vascular changes. We used the Cstatistic and NRI to access and compare the effects of models. Our study also has a few limitations. The cross-sectional nature of the study would not clarify the causal relationship; therefore, a larger cohort in the future is required. A few subjects were not enrolled in the study because of noncompliance, leading to a possible selection bias. The use of a questionnaire is another limitation, which may cause recall bias. In addition, our results may not be generalized to other ethnicities, because the study was only validated in Chinese residents.

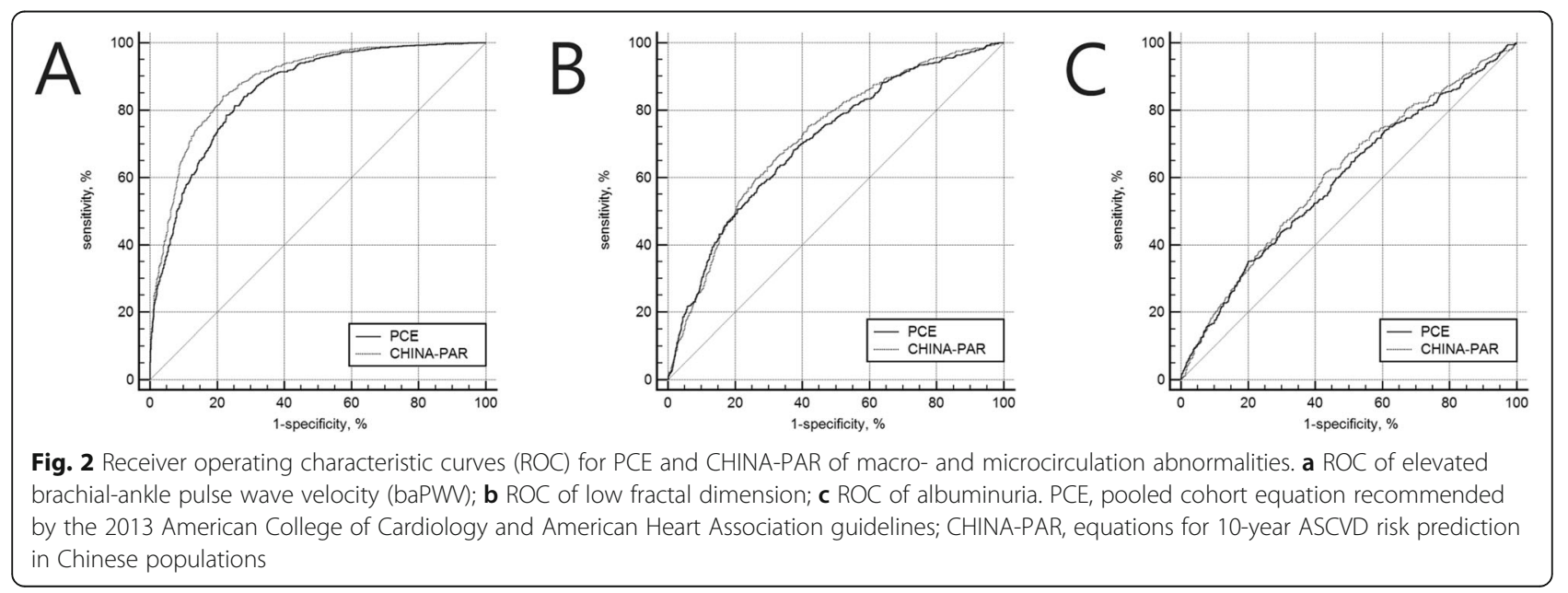




\section{Conclusion}

Overall, our study demonstrated that the CHINA-PAR equations rather than PCE could provide better identification of macro- and microcirculation abnormalities. A lower cut-off point for subclinical vascular changes may be selected in a population from southeast China.

\section{Supplementary information}

Supplementary information accompanies this paper at https://doi.org/10. 1186/s12872-020-01425-0.

Additional file 1: Supplemental Table 1. comparison of CHINA-PAR

and PCE models

\section{Abbreviations}

CVD: Cardiovascular disease; PCE: Pooled cohort equations;

ASCVD: Atherosclerotic CVD; CHINA-PAR: Prediction for ASCVD risk in China project; baPWV: Brachial-ankle pulse wave velocity; TC: Total cholesterol; HDLC: High-density lipoprotein cholesterol; LDL-C: Low-density lipoprotein cholesterol; HbA1c: Glycosylated hemoglobin; BMl: Body mass index; UACR: Urine albumin to creatinine ratio; eGFR: The estimated glomerular filtration rate; ROC: Receiver operating characteristic; NRI: Net reclassification improvement

\section{Acknowledgements}

Not applicable.

\section{Authors' contributions}

QW Li analyzed and interpreted the participant data as a major contributor in writing the manuscript. F Lin has been involved in drafting the manuscript or revising it critically for important intellectual content. ZH Gao was responsible for the fundus photography and analysis of retinal vessel parameters. F Huang have made contributions to the design and execution of the work, and she was in charge of acquisition, analysis and interpretation of data. PL Zhu made substantial contributions to conception and design. He is responsible for the whole study. Each author has participated sufficiently in the work to take public responsibility for appropriate portions of the content, and agreed to be accountable for all aspects of the work. The author(s) read and approved the final manuscript.

\section{Funding}

The study was supported by Startup Fund for Scientific Research from Fujian Medical University (grant number: 2016QH122). The funding body had no role in study design, data collection and analysis and in writing the manuscript.

\section{Availability of data and materials}

The datasets generated and analyzed during the current study are not publicly available due to the fact that the study is still followed up but are available from the corresponding author on reasonable request.

\section{Ethics approval and consent to participate}

This study was performed according to the World Medical Association Declaration of Helsinki and approved by the Ethics Committee of the Fujian Provincial Hospital. A written informed consent was obtained from all individuals.

\section{Consent for publication}

Not applicable.

\section{Competing interests}

The authors declare that they have no competing interests.

\section{Author details}

${ }^{1}$ Department of Geriatric Medicine, Fujian Provincial Hospital, Fujian Provincial Center for Geriatrics, Fujian Provincial Key Laboratory of Geriatric Disease, Shengli Clinical Medical College of Fujian Medical University, 134 East Street, Fuzhou 350001, Fujian, China. '2Department of ophthalmology, Fujian Provincial Hospital, Shengli Clinical Medical College of Fujian Medical University, Fuzhou, China.
Received: 24 May 2019 Accepted: 12 March 2020

Published online: 24 March 2020

\section{References}

1. Shiwei $L$, Yichong $L$, Xinying $Z$, Haidong W, Peng Y, Lijun W, et al. Burden of Cardiovascular Diseases in China, 1990-2016: findings from the 2016 global burden of disease study. JAMA Cardiol. 2019, 4:342-52

2. Benjamin EJ, Muntner P, Alonso A, Bittencourt MS, Callaway CW, Carson AP, et al. Heart Disease and stroke statistics-2019 update. Circulation. 2019;139: e56-e528.

3. Goff DC, Lloyd-Jones DM, Bennett G, Coady S, D'agostino RB, Gibbons R, et al. 2013 ACC/AHA guideline on the assessment of cardiovascular risk: a report of the American College of Cardiology/American Heart Association Task Force On Practice Guidelines. J Am Coll Cardiol. 2014;63(25 Part B): 2935-59.

4. Yang X, Li J, Hu D, Chen J, Li Y, Huang J, Liu X, Liu F, Cao J, Shen C. Predicting the 10-year risks of atherosclerotic cardiovascular disease in Chinese population: the China-PAR project (prediction for ASCVD risk in China). Circulation. 2016;134(19):1430-40.

5. Tang $X$, Zhang D, He L, Wu N, Si Y, Cao Y, et al. Performance of atherosclerotic cardiovascular risk prediction models in a rural northern Chinese population: results from the Fangshan cohort study. Am Heart J. 2019;211:34-44.

6. Zeng NM, Zheng XW, Peng $H$, Jiao $Y$, Li HM, Zhang MZ, Wang AL, Zhang $\mathrm{YH}$. Validation of the China-PAR equations for cardio-cerebrovascular risk prediction in the inner Mongolian population. Biomed Environ Sci. 2018; 31(6):463-6.

7. Sugawara J, Tanaka H. Brachial-ankle pulse wave velocity: myths, misconceptions, and realities. Pulse (Basel). 2015;3(2):106-13.

8. Kawasaki R, Che Azemin MZ, Kumar DK, Tan AG, Liew G, Wong TY, Mitchell $P$, Wang JJ. Fractal dimension of the retinal vasculature and risk of stroke: a nested case-control study. Neurology. 2011;76(20):1766-7.

9. Shah SJ, Lam CSP, Svedlund S, Saraste A, Hage C, Tan RS, et al. Prevalence and correlates of coronary microvascular dysfunction in heart failure with preserved ejection fraction: PROMIS-HFpEF. Eur Heart J. 2018;39(37):3439-50.

10. Cheng $\mathrm{YH}$, Cheng HS, Tarng DC. More impact of microalbuminuria on retinopathy than moderately reduced GFR among type 2 diabetic patients. Diabetes Care. 2012;35(4):803-8.

11. Aguilar MI, O'Meara ES, Seliger S, Longstreth WT, Hart RG, Pergola PE, et al. Albuminuria and the risk of incident stroke and stroke types in older adults. Neurology. 2010;75(15):1343-50.

12. Furtner M, Kiechl S, Mair A, Seppi K, Weger S, Oberhollenzer F, Poewe W, Willeit J. Urinary albumin excretion is independently associated with carotid and femoral artery atherosclerosis in the general population. Eur Heart J. 2005;26(3):279-87

13. Levey AS, Stevens LA, Schmid CH, Zhang YL, Castro AF, Feldman HI, et al. A new equation to estimate glomerular filtration rate. Ann Intern Med. 2009; 150(9):604-12.

14. Kidney Disease. Improving Global Outcomes (KDIGO) CKD Work Group. KDIGO 2012 clinical practice guideline for the evaluation and management of chronic kidney disease. Kidney Int Suppl. 2013;3:1-150.

15. Chobanian AV, Bakris GL, Black HR, Cushman WC, Green LA, Izzo JL Jr, et al. Seventh report of the joint national committee on prevention, detection, evaluation, and treatment of high blood pressure. Hypertension. 2003;42(6): 1206-52.

16. Sonne DP, Hemmingsen B. Comment on American Diabetes Association. Standards of medical Care in Diabetes-2017. Diabetes care 2017; 40 (Suppl. 1): S1-S135. Diabetes Care 2017;40(7):e92-e93.

17. Yamashina A, Tomiyama H, Arai T, Hirose K, Koji Y, Hirayama Y, Yamamoto Y, Hori S. Brachial-ankle pulse wave velocity as a marker of atherosclerotic vascular damage and cardiovascular risk. Hypertens Res. 2003;26(8):615-22.

18. Ciccone MM, Bilianou E, Balbarini A, Gesualdo M, Ghiadoni L, Metra M, et al. Task force on: 'Early markers of atherosclerosis: influence of age and sex'. J Cardiovasc Med (Hagerstown). 2013;14(10):757-66.

19. Reference Values for Arterial Stiffness Collaboration. Determinants of pulse wave velocity in healthy people and in the presence of cardiovascular risk factors: 'establishing normal and reference values'. Eur Heart J. 2010;31(19): 2338-50.

20. Kipli K, Hoque ME, Lim LT, Mahmood MH, Sahari SK, Sapawi R, Rajaee N, Jpseph A. A review on the extraction of quantitative retinal microvascular image feature. Comput Math Methods Med. 2018;2018:1-21. 
21. Cheung CY, Ong YT, Ikram MK, Ong SY, Li X, Hilal S, et al. Microvascular network alterations in the retina of patients with Alzheimer's disease. Alzheimer Dement. 2014;10(2):135-42.

22. Wang SB, Mitchell P, Liew G, Wong TY, Phan K, Thiagalingam A, Joachim N, Burlutsky $G$. A spectrum of retinal vasculature measures and coronary artery disease. Atherosclerosis. 2018;268:215-24.

23. Liew G, Mitchell P, Rochtchina E, Wong TY, Hsu W, Lee ML, Wainwright A, Wang JJ. Fractal analysis of retinal microvasculature and coronary heart disease mortality. Eur Heart J. 2011;32(4):422-9.

24. Topel ML, Shen J, Morris AA, Al Mheid I, Sher S, Dunbar SB, et al. Comparisons of the Framingham and pooled cohort equation risk scores for detecting subclinical vascular Disease in blacks versus whites. Am J Cardiol. 2018;121(5):564-9.

\section{Publisher's Note}

Springer Nature remains neutral with regard to jurisdictional claims in published maps and institutional affiliations.

Ready to submit your research? Choose BMC and benefit from:

- fast, convenient online submission

- thorough peer review by experienced researchers in your field

- rapid publication on acceptance

- support for research data, including large and complex data types

- gold Open Access which fosters wider collaboration and increased citations

- maximum visibility for your research: over $100 \mathrm{M}$ website views per year

At BMC, research is always in progress.

Learn more biomedcentral.com/submissions 